\title{
TAGUNG
}

\section{Internationale Korruptionsbekämpfung auf dem Prüfstand}

\author{
Sebastian Wolf und Diana Schmidt-Pfister*
}

Noch in den späten 1980er Jahren wäre es vermutlich kaum möglich gewesen, eine ganze wissenschaftliche Tagung dem Thema ,internationale Korruptionsbekämpfung ‘ zu widmen. Jahrzehntelang war die Eindämmung der Korruption für Praktiker und Wissenschaftler - wenn überhaupt - ein rein nationales Thema. Erst seit Mitte der 1990er Jahre, nach dem Ende des Ost-West-Konflikts, mit dem Aufkommen neuer zivilgesellschaftlicher Akteure und im Zuge der Globalisierung begann die transnationale Korruptionsbekämpfung einen wichtigen Stellenwert auf der Agenda verschiedener internationaler Organisationen einzunehmen. Seitdem erlebt das Thema einen beeindruckenden Boom.

Doch die tatsächliche Implementation der verschiedenen Instrumente steckt noch in den Anfängen. Zudem bleibt das Feld der Antikorruptionsforschung stark fragmentiert. Die Tagung „International Anti-Corruption Regimes in Europe" hatte es sich vor diesem Hintergrund zur Aufgabe gemacht, von einem interdisziplinären Standpunkt aus die letzten 10 bis 20 Jahre der internationalen Korruptionsbekämpfung mit einem geografischen Schwerpunkt auf Europa zu reflektieren und neueste Forschungsergebnisse sowie Einschätzungen von Praktikern zu diskutieren. Im Folgenden werden die Beiträge und $\mathrm{Er}$ gebnisse der Tagung gegliedert nach den übergeordneten Bereichen, Governance internationaler Antikorruptionsregime', ,Erfolge und Misserfolge der internationalen Korrupti-

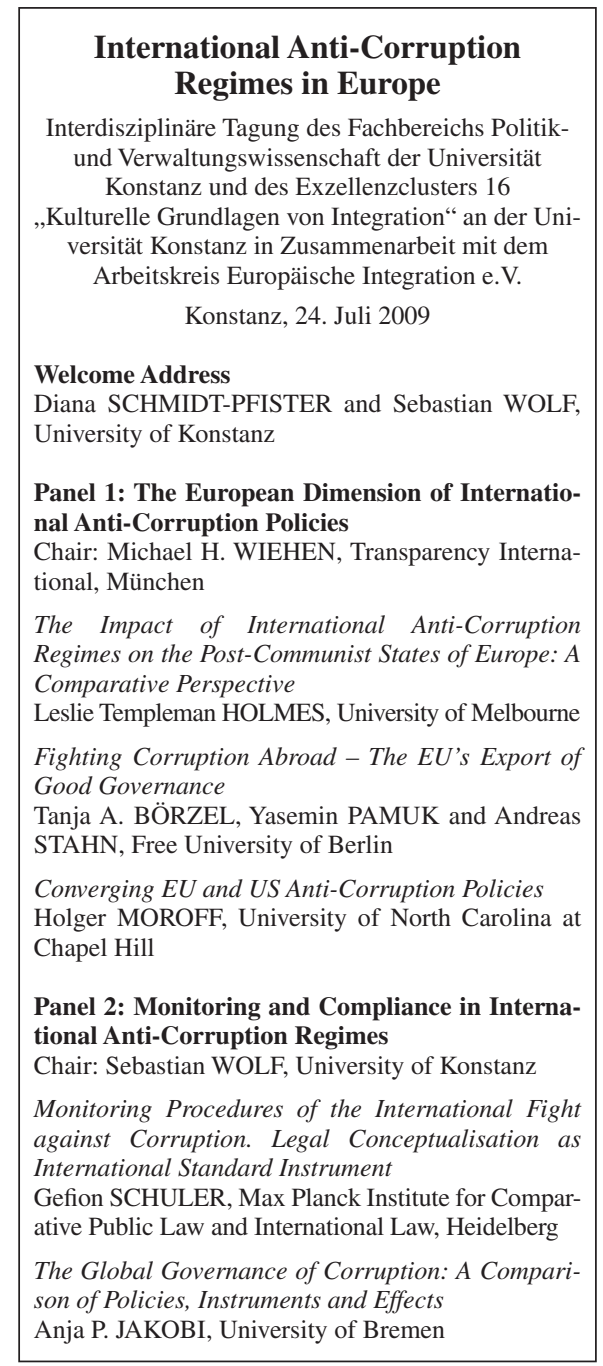

* Dr. Sebastian Wolf, wissenschaftlicher Mitarbeiter am Fachbereich Politik- und Verwaltungswissenschaft der Universität Konstanz.

Dr. Diana Schmidt-Pfister, wissenschaftliche Mitarbeiterin im Exzellenzcluster 16,„Kulturelle Grundlagen von Integration" an der Universität Konstanz.

Die überarbeiteten Tagungsbeiträge werden 2010 in einem Sammelband mit dem Titel „International AntiCorruption Regimes in Europe: Between Corruption, Integration, and Culture" veröffentlicht. 
onsbekämpfung ' und ,Zukunftsperspektiven internationaler Antikorruptionsmaßnahmen " zusammengefasst und aufeinander bezogen.

\section{Governance internationaler Antikorruptions- regime}

Hilfreiche Überblicksdarstellungen über Aufbau und Regelungsstrukturen verschiedener Antikorruptionsregime lieferten vor allem die Beiträge von Leslie Templeman Holmes und Anja P. Jakobi. Hier wurden in aller Kürze, aber unter Herausarbeitung der jeweiligen Besonderheiten, die Antikorruptionspolitiken der Europäischen Union, des Europarats, der Organisation für wirtschaftliche Zusammenarbeit und Entwicklung (OECD), der Vereinten Nationen (VN) und der Weltbank skizziert. Einen Schwerpunkt legte der von der Regime-Theorie der internationalen Beziehungen inspirierte Beitrag von Jakobi auf die Evaluierungsgremien der verschiedenen internationalen Antikorruptionsregime. Dies war auch das zentrale Thema des Vortrags von Gefion Schuler, die sich im Detail mit den rechtlichen Implikationen des Monitoringmechanismus der OECD-Arbeitsgruppe zur Bekämpfung der Bestechung im internationalen Geschäftsverkehr auseinandersetzte. Georg Huber-Grabenwarter präsentierte eine gute Einführung in die vielfältigen Ansätze der VN-Konvention gegen Korruption. Demgegenüber behandelten Tanja A. Börzel, Yasemin Pamuk und Andreas Stahn in ihrem Beitrag den Charakter der unterschiedlichen externen EU-Politiken zur Verbreitung von ,good governance ' in Drittstaaten. Der praxisorientierte Vortrag von Bryane Michael und Donald Bowser skizzierte die Merkmale verschiedener Wellen der zwischenstaatlichen Kooperation bei der Korruptionsbekämpfung.

Es wurde deutlich, dass die internationalen Bestrebungen zur Eindämmung der Korruption in den letzten circa 15 Jahren eine Vielzahl sich teilweise ergänzender und zum Teil überlappender internationaler Antikorruptionsregime hervorgebracht haben. Divergenzen gibt es insbesondere hinsichtlich der Zahl und Heterogenität der Mitgliedstaaten, der be-
Panel 3: Cultural Perceptions of Corruption and International Anti-Corruption Strategies

Chair: Diana SCHMIDT-PFISTER, University of Konstanz

The Gap Between Anti-Corruption Measures and Public Perceptions of Corruption: Meeting the Challenge in the EU-Research Projects "Crime and Culture" and "ALACs"

Dirk TÄNZLER, University of Konstanz, Angelos GIANNAKOPOULOS, University of Konstanz and Ben ELERS, Transparency International, Berlin

Culture, Corruption and Anti-Corruption Strategies in Post-Communist Europe

Åse Berit GRØDELAND, Chr. Michelsen Institute/ Norwegian Institute for Urban and Regional Research, Bergen/Oslo

Roundtable discussion: Practitioners' Experiences in Fighting Corruption in Europe and Beyond

Chair: Michael H. WIEHEN, Transparency International, München

The Evolution of the Anti-Corruption Industry in the Third Wave of Anti-Corruption Work

Bryane MICHAEL, Linacre College, University of Oxford and Donald BOWSER, University of Melbourne

Mismatches Between Anti-Corruption Measures and the Public Perception of Corruption

Anne LUGON-MOULIN, Basel Institute on Governance, Basel

Supporting the Implementation of International Anti-Corruption Initiatives: Opportunities and Limitations for Technical Assistance

Georg HUBER-GRABENWARTER, German UNCAC Project, Deutsche Gesellschaft für Technische Zusammenarbeit, Eschborn

handelten Korruptionsphänomene, der Verbindlichkeit und Reichweite der Maßnahmen sowie der Ausgestaltung der Evaluierungsverfahren. Vor allem Holger Moroff und HuberGrabenwarter konstatierten eine zunehmende Depolitisierung der Korruptionsbekämpfung. Nach der Verabschiedung internationaler Antikorruptionsinstrumente sind die nationalen und internationalen Verwaltungen mit der Implementierung betraut. Sie entwickeln die Rechtsinstrumente mitunter dynamisch und extensiv weiter, wie Schuler bemerkte. Dies kann zwar aus der Perspektive der Korruptionsbekämpfung vorteilhaft, demokratietheoretisch aber auch bedenklich sein. 
Erfolge und Misserfolge der internationalen Korruptionsbekämpfung

Nahezu alle Beiträge beleuchteten sowohl positive als auch negative Entwicklungen der letzten Jahre. Was die Erfolge anbelangt, so kam Holmes in seiner vergleichenden Analyse zu dem Ergebnis, die EU-Beitrittspolitik habe im Wesentlichen günstige Auswirkungen auf die Antikorruptionsperformanz der betreffenden Staaten in Mittel- und Osteuropa gezeigt. Börzel, Pamuk und Stahn bewerteten die EU-Ansätze zur Förderung von ,good governance ' in Drittstaaten grundsätzlich positiv. Auch fanden die Europäische Union und die USA, wie in Moroffs Diskursanalyse nachgezeichnet, trotz ursprünglich unterschiedlicher Korruptionsbekämpfungskonzeptionen schließlich zu einem gemeinsamen Ansatz zur Kriminalisierung transnationaler Korruption. Jakobi wie auch Schuler bewerteten die vergleichsweise strengen Monitoringverfahren von Europarat und OECD positiv. Die VN-Konvention gegen Korruption stellt laut Huber-Grabenwarter einen sehr nützlichen Ausgangspunkt für technische Zusammenarbeit und Entwicklungshilfe zur Bekämpfung von Korruption dar. Anne LugonMoulin wies unter anderem auf die nachhaltige Wirkung zahlreicher Korruptions-Sensibilisierungskampagnen der letzten Jahre hin. Dirk Tänzler, Angelos Giannakopoulos und Ben Elers berichteten über ein vielversprechendes Projekt zum Aufbau von (Rechts-) Beratungszentren in Mittel- und Osteuropa, die Bürger konkret bei der Prävention und Bekämpfung von Korruption unterstützen sollen und dies vielerorts bereits tun. Insgesamt, so Michael und Bowser, sei eine positive Entwicklung weg von symbolischen Konferenzen und oberflächlichen Studien hin zu praxisrelevanter internationaler Kooperation bei der Verfolgung von Korruptionsfällen zu konstatieren.

Auch negative Entwicklungen der internationalen Initiativen zur Korruptionsbekämpfung wurden ausführlich diskutiert. So verwies etwa Holmes auf die relativ schwache Anti- korruptionsperformanz der postsozialistischen Länder ohne EU-Beitrittsperspektive. Nach Ansicht von Börzel, Pamuk und Stahn könnte die input-Dimension der EU-Politik zur externen Förderung von, good governance" noch stärker gefördert werden. Jakobi wie auch Schuler thematisierten Defizite in den Antikorruptions-Evaluierungsverfahren beispielsweise auf Ebene der Afrikanischen Union und der Vereinten Nationen. Tänzler, Elers und Giannakopoulos wiesen auf die begrenzte Wirkung bestimmter nationaler Korruptionsbekämpfungsmaßnahmen in Osteuropa aufgrund von Diskrepanzen zwischen den Korruptionsperzeptionen in der Bevölkerung und der Ausrichtung der präventiven und repressiven Instrumente hin. Åse Berit Grødeland stellte die Ergebnisse umfangreicher Befragungen zu korruptionsbegünstigenden Einstellungen in mehreren osteuropäischen Ländern vor, welche die ungebrochene, aber in internationalen Regimen oft vernachlässigte Präsenz kulturell bedingter Traditionslinien und informeller Praktiken aufzeigen. Michael und Bowser skizzierten, wie sich die internationale Korruptionsbekämpfung in den letzten Jahren zu einer regelrechten Industrie mit teilweise zweifelhaften, stark verselbstständigten Mechanismen entwickelt habe. Beträchtliche Unterschiede zwischen Korruptionswahrnehmungsindizes und -opferindizes, die eine Überschätzung der Korruption in bestimmten Sektoren implizieren, sind nach Ansicht von Lugon-Moulin eine negative Folge der zahlreichen Sensibilisierungsmaßnahmen. Huber-Grabenwarter beschrieb Schwierigkeiten bei der technischen Zusammenarbeit im Antikorruptionssektor unter anderem aufgrund politischer Blockaden, kultureller Hürden und interner Interessenkonflikte in den Zielländern.

\section{Zukunftsperspektiven internationaler Antikor- ruptionsmaßnahmen}

Die Wirtschafts- und Finanzkrise könnte nach Ansicht von Sebastian Wolf zu mehr deviantem Verhalten im Allgemeinen und Korruption im Speziellen führen, um Aufträge und 
Arbeitsplätze im globalisierten Wettbewerb zu erhalten. Hier stehen internationale Antikorruptionsregime vor der Aufgabe, erreichte Erfolge zu sichern und bestehende Defizite nicht größer werden zu lassen. Erfolgreiche Patentrezepte hat es in der Korruptionsbekämpfung zwar nie gegeben. Dennoch lassen sich aus den Tagungsbeiträgen bei aller $\mathrm{Zu}$ rückhaltung einige Handlungsempfehlungen und Zukunftsperspektiven ableiten. Folgt man Holmes, so sollte die EU-Beitrittspolitik wohl auch weiterhin - und vielleicht noch in größerem Umfang - Konditionierungspolitik sein. Damit bleibt aber immer noch das Problem, dass die Europäische Union in Sachen Korruptionsbekämpfung zu nachlässig mit ihren alten Mitgliedstaaten umgeht und es derzeit eine gegen Grundprinzipien der Union verstoBende Ungleichbehandlung von alten, neuen und potenziellen EU-Mitgliedern gibt. Den Ausführungen von Börzel, Pamuk und Stahn lässt sich entnehmen, dass die externe, good governance'-Politik der Europäischen Union noch kohärenter gestaltet und stärker auf Korruptionsbekämpfung im engeren Sinne ausgerichtet werden könnte. Moroffs Diskursanalyse kann so interpretiert werden, dass sich die traditionell input-orientierte US-amerikanische Debatte über Korruption und der bislang vorwiegend output-orientierte europäische Korruptionsdiskurs künftig stärker wechselseitig befruchten sollten.

Jakobi wie auch Schuler haben auf die Notwendigkeit hingewiesen, internationale Antikorruptionsregime mit effektiven Evaluierungsgremien und -verfahren auszustatten. Die jüngsten Entwicklungen auf VN-Ebene stimmen hier eher pessimistisch. Michael $H$. Wiehen machte auf das vergleichsweise effektive Mittel der Weltbank aufmerksam, Unternehmen bei Korruptionsverdachtsfällen von ihren Projekten ausschließen zu können, ohne an die hohen Beweishürden in nationalen Strafprozessen gebunden zu sein. Begrüßenswert erscheint auch die geplante und teilweise bereits erfolgte Einrichtung bürgerorientierter Antikorruptionszentren in verschiedenen mittel- und osteuropäischen Ländern, die unter Berücksichtigung der von Grødeland sowie Tänzler, Elers und Giannakopoulos geschilderten, kulturell unterschiedlichen Perzeptionen von Korruption und Korruptionsbekämpfung kontext-spezifische Lösungen entwickeln könnten. Aus Lugon-Moulins Beitrag lässt sich die Folgerung entnehmen, anstelle von allgemeinen Sensibilisierungskampagnen lieber verstärkt auf konkrete und vor allem kriminalisierende Maßnahmen zur Korruptionsprävention zu setzen. Es leuchtet ein, dass die technische Zusammenarbeit und Entwicklungshilfe bei der Korruptionsbekämpfung trotz der von Huber-Grabenwarter dargestellten Probleme in Zukunft fortgesetzt werden sollte. Im EU-Kontext scheinen insbesondere Twinning-Programme mit dem Austausch von Verwaltungsspezialisten positive Wirkung zu entfalten. Schließlich muss die internationale Korruptionsbekämpfung angesichts der Vielzahl und Komplexität von Regelungsstrukturen, Projekten, Finanzmitteln und involvierten Akteuren darauf achten, sich nicht - wie Michael und Bowser dies andeuteten - zu einem selbstreferentiellen System zu entwickeln. 